\section{Polymeric Composite Dressings Containing Calcium-Releasing Nanoparticles Accelerate Wound Healing in Diabetic Mice}

\author{
AU1 Soledad Perez-Amodio, ${ }^{1-3}$ Nuria Rubio, ${ }^{1,4}$ Olaia F Vila, ${ }^{1,4}$ \\ Claudia Navarro-Requena, ${ }^{1,2}$ Oscar Castaño, $1,2,5,6$ \\ Aitor Sanchez-Ferrero, ${ }^{1,2}$ Joan Marti-Munoz,, ${ }^{1,2}$ Mercè Alsina-Gibert, ${ }^{7}$ \\ Jeronimo Blanco, ${ }^{1,4}$ and Elisabeth Engel ${ }^{1-3, *}$

\footnotetext{
${ }^{1}$ Biomedical Research Networking Center in Bioengineering, Biomaterials, and Nanomedicine (CIBER-BBN), Madrid, Spain ${ }^{2}$ Institute for Bioengineering of Catalonia (IBEC), The Barcelona Institute of Science and Technology, Barcelona, Spain. ${ }^{3}$ Materials Science and Metallurgical Engineering, Polytechnic University of Catalonia (UPC), Barcelona, Spain.

${ }^{5}$ Electronics and Biomedical Engineering, Universitat de Barcelona (UB), Barcelona, Spain.

${ }^{6}$ Bioelectronics Unit and Nanobioengineering Lab., Institute for Nanoscience and Nanotechnology of the University of Barcelona (IN2UB), Barcelona, Spain.

${ }^{7}$ Department of Dermatology, Hospital Clinic de Barcelona, Universitat de Barcelona, Barcelona, Spain.
} \\ ${ }^{4}$ Catalonian Institute for Advanced Chemistry (IQAC-CSIC), Barcelona, Spain.
}

Objective: Wound healing is a complex process that involves the interaction between different cell types and bioactive factors. Impaired wound healing is characterized by a loss in synchronization of these interactions, resulting in nonhealing chronic wounds. Chronic wounds are a socioeconomic burden, one of the most prominent clinical manifestations of diabetes, however, they lack satisfactory treatment options. The objective of this study was to develop polymeric composites that deliver ions having wound healing properties and evaluate its performance using a pressure ulcer model in diabetic mice.

Approach: To develop a polymeric composite wound dressing containing ionreleasing nanoparticles for chronic wound healing. This composite was chemically and physically characterized and evaluated using a pressure ulcer wound model in diabetic $(\mathrm{db} / \mathrm{db})$ mice to explore their potential as novel wound dressing.

Results: This dressing exhibits a controlled ion release and a good in vitro bioactivity. The polymeric composite dressing treatment stimulates angiogenesis, collagen synthesis, granulation tissue formation, and accelerates wound closure of ischemic wounds created in diabetic mice. In addition, the performance of the newly designed composite is remarkably better than a commercially available dressing frequently used for the treatment of lowexuding chronic wounds.

Innovation: The developed nanoplatforms are cell- and growth factor free and control the host microenvironment resulting in enhanced wound healing. These nanoplatforms are available by cost-effective synthesis with a defined composition, offering an additional advantage in potential clinical application. Conclusion: Based on the obtained results, these polymeric composites offer an optimum approach for chronic wound healing without adding cells or external biological factors.

Keywords: chronic wounds, bioactive dressings, diabetes, angiogenesis

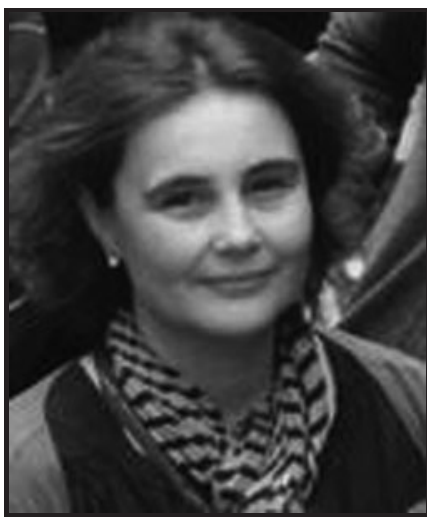

Elisabeth Engel, PhD

Submitted for publication April 17, 2020. Accepted in revised form June 12, 2020.

${ }^{*}$ Correspondence: Biomaterials for Regenerative Therapies Group, Institute for Bioengineering of Catalonia, The Barcelona Institute of Science and Technology, Ed. Hèlix, Baldiri Reixac 15-21, Barcelona 08028

(e-mail: eengel@ibecbarcelona.eu). 


\section{INTRODUCTION}

DiAbetes mellitus has become a major global health epidemic with a particularly large incidence in developed countries. A study recently reported in Diabetes Research and Clinical Practice, estimated that by 2035, the number of diabetesaffected people would reach at least 592 million people worldwide. ${ }^{1}$ Approximately $25 \%$ of these patients will develop diabetic foot ulcer (DFU) that results from peripheral neuropathy and impaired wound healing (characterized by chronic inflammation, impaired angiogenesis, and decreased collagen production). DFUs lead to frequent hospitalization and in extreme cases, to amputations that result in elevated hospital costs and poor quality of life for patients. ${ }^{2}$

Therefore, considerable efforts have been made to effectively promote wound healing and skin regeneration of chronic wounds. ${ }^{3}$ As part of the many efforts to accelerate the wound healing process of chronic wounds, several studies have focused on the development of biological based therapies, such as growth factor administration and bioengineered skin substitutes. ${ }^{4}$

Despite all the advances reported using skin substitutes and exogenous growth factors in a wound healing context, translation of these in the clinical settings is still reduced. Major barriers to clinical translation include regulatory, economic, and safety issues. ${ }^{5}$

Bioceramics have been investigated and used in applications toward bone and teeth regeneration. However, these biomaterials have been recently acknowledged for their healing capacity and potential to promote the regeneration of new blood vessels in soft tissue. ${ }^{6}$ Indeed, several studies reported on accelerated wound healing when applying composite materials containing bioactive glasses on skin wounds. ${ }^{7-9}$ Degradation of bioceramics results in the release of soluble ionic products that seem to strongly affect cell behavior. ${ }^{10-12}$

Among other ionic products, calcium is released from most bioceramics. Calcium, as reported in several in vitro and in vivo studies, plays an important role in all phases of wound healing, even though the best acknowledged of these is its implication in the clotting cascade. ${ }^{13}$ In vitro studies have shown that calcium stimulates proliferation and migration of keratinocytes. ${ }^{14,15}$ In addition, dermal fibroblast behavior has been reported to be affected by extracellular calcium. Although the effect of calcium on fibroblast proliferation, collagen synthesis, and cell morphology has been demonstrated, ${ }^{16-18}$ concentration ranges of this ion af- fecting these events are still unknown. Recently, our group demonstrated that extracellular calcium at concentrations of 2.5 and $3.5 \mathrm{mM}$ increased metabolic activity, in vitro wound closure, matrix metalloproteinases activity, collagen synthesis and cytokine expression, and reduced cell contraction capacity on rat dermal fibroblasts. ${ }^{18}$ This study also reported the synthesis of calcium phosphatebased ormoglass nanoparticles, whose ionic dissolution produces a similar stimulating effect as extracellular calcium on dermal fibroblasts. ${ }^{18}$

Calcium not only promotes wound healing responses in keratinocytes and fibroblasts, but this ion has been described as a stimulator of angiogenesis. We have demonstrated that the increased local concentration of calcium $(\mathrm{Ca})$ ions could induce the migration, maturation, and reorganization in a tubular network-like structure of endothelial progenitor cells. ${ }^{19}$ In addition, a previous study has revealed that calcium-releasing composite biomaterials promoted angiogenesis using in vitro and in vivo models. ${ }^{20}$ It is well known that angiogenesis plays an essential role in wound healing, especially in nonhealing diabetic wounds, where the formation of new blood vessels is compromised. ${ }^{21}$

Based on previously obtained results, in the present study, we fabricated poly(lactic acid) (PLA) fiber matrices loaded with calcium-releasing nanoparticles (SG5) (PLA-SG5) ${ }^{18}$ and explored their healing effects on pressure ulcer wounds in diabetic mice Nonofibrous PLA platforms have wide applicabil $\bigcirc$ t only as scaffolds for tissue regeneration, but also as drug delivery vehicles. ${ }^{22}$ Therefore, it was hypothesized that PLA-SG5 mats would effectively promote the healing of pressure ulcer wounds through the effects of calcium. The physicochemical and mechanical properties of PLA and PLA-SG5 mats were determined and the calcium release behavior was examined. Furthermore, the in vivo wound healing capacity was investigated using a pressure ulcer wound model in diabetic $(\mathrm{db} / \mathrm{db})$ mice to explore their potential as novel wound dressing.

\section{CLINICAL PROBLEM ADDRESSED}

Chronic wounds have a huge economic impact in developed countries, and it is expected to rise as the population ages. Current therapies cannot fully address the impaired healing, provoking wound complications, such as infections and poor wound closure, therefore, new technologies are needed.

We have developed a cost-effective new polymeric composite wound dressing that offers an 
optimum approach for wound healing without adding cells or external biological factors.

\section{MATERIALS AND METHODS}

\section{Preparation of PLA and PLA-SG5 mats}

Calcium phosphate ormoglass particles referred as SG5 with a composition of $\mathrm{CaO}: \mathrm{P}_{2} \mathrm{O}_{5}: \mathrm{Na}_{2} \mathrm{O}: \mathrm{TiO}_{2}$ (44.5:44.5:6:5) were synthesized by the sol-gel method using metal alkoxides, as previously reported. ${ }^{23}$ The PLA and PLA nanofiber mats containing 25\% (w/w) of the SG5 nanoparticles (PLA and PLA-SG5 mats) were manufactured with the electrospinning technique as explained elsewhere. ${ }^{20}$ The process of electrospinning is a widely used method to produce biomaterials and allows to generate extracellular matrix-like mats of polymer nanofibers. It consists of applying a high voltage into a polymer solution to increase the electrostatic repulsion between the polymer molecules and thus achieve nanofibers of the polymer. ${ }^{24}$

To generate the PLA and PLA-SG5 nanofiber mats, we dispersed the right amount of the SG5 nanoparticles to have a final concentration of a $25 \%$ $(\mathrm{w} / \mathrm{w})$ in $15 \mathrm{~mL}$ of 2,2,2-trifluoroethanol (TFE) (99.8\%; Panreac). The nanoparticles were sonicated using a Branson Ultrasonic 450W Sonifier Model102C (Branson Ultrasonics, Danbury, CT). Then, PLA pellets (70/30 L-lactide/DL-lactide copolymer, Purasorb PLDL 7038) to have a final concentration of $4 \%(\mathrm{w} / \mathrm{w})$ with respect to the $\mathrm{TFE}$, were added under vigorous stirring. After $\sim 3 \mathrm{~h}$ of stirring at room temperature (RT), the polymer solution was transferred into a polypropylene/ polystyrene sterilized syringe with a 23GA tip (Precision tips, Nordson EFD). The electrospinning process was performed at $10 \mathrm{kV}$ of applied voltage, $1 \mathrm{~mL} / \mathrm{h}$ dispensing pump rate, and $15 \mathrm{~cm}$ tipcollector distance. Nanofibers were deposited on aluminum foil placed on a rotatory grounded collector spinning at 1,200 rpm to obtain an aligned fiber orientation. Before the electrospinning process, we covered the aluminum foil with a thin glycerol layer (99\%; Panreac) to facilitate the detachment of the deposited nanofibers. Control mats consisting of pure PLA nanofibers, were manufactured under the same conditions avoiding the particles. Once synthesized, the nanofiber mats were kept in a desiccator to improve its preservation and to allow the evaporation of possible cytotoxic TFE traces.

For pore architecture analysis, the nanofiber mats were rendered fluorescent by dissolving a $0.01 \%(\mathrm{w} / \mathrm{w})$ of Rhodamine B dye in the polymer solution before the electrospinning process. At this concentration, we did not observe apparent effects in the morphology of the nanofiber mats or its cytotoxicity.

\section{Morphological characterization of mats}

Field emission scanning electron microscope characterization. Micro and nanomorphology of PLA and PLA-SG5 mats were characterized by using an Ultra-High-Resolution Field Emission Scanning Electron Microscopy (FE-SEM, Nova ${ }^{\mathrm{TM}}$ Nano SEM-230; FEI Co., Hillsboro, OR) operating at $5.00 \mathrm{kV}$. Before imaging, samples were coated with an ultrathin carbon layer to improve conductivity. Mean fiber diameter was measured considering at least 25 randomly selected fibers and using the ImageJ analysis software. ${ }^{25}$

Water contact angle. Wettability of the samples was evaluated by measuring the static contact angle with a video-based optical contact angle measuring device (OCA 15 plus from Data Physics Instruments) using the sessile drop technique. The selected water droplet volume was $3 \mu \mathrm{L}$.

Pore architecture. The porosity of the PLA and PLA-SG5 nanofiber mats was characterized by confocal laser scanning microscopy (CLSM) imaging. PLA-rhodamine and PLA-SG5-rhodamine mats were placed between slides and coverslips using Mowiol and kept overnight at $4^{\circ} \mathrm{C}$ to allow the mounting medium to harden. Samples were then visualized with a Leica TCS SP5 confocal microscope (Leica Microsystem, Wetzlar, Germany) to a total depth of $30 \mu \mathrm{m}$ with a step size of $0.29 \mu \mathrm{m}$, using a HCX PL APO lambda blue $63.0 \times 1.40$ OIL UV objective (Leica), at an excitation wavelength of $561 \mathrm{~nm}$ (with a 26\% laser power) and an emission wavelength range of 579-657 nm. A double AOTFPMT (acousto-optic tunable filter-photomultiplier tube) compensation was used to ensure a constant mean intensity through the $z$ planes. Threedimensiomal reconstructions were generated using Volocity software. The mean pore area and the mean area percentage occupied by the pores were calculated using the Fiji software using the Analyze Particles plugin. ${ }^{25}$ Briefly, three PLA-Rho and three PLA-SG5-Rho samples were analyzed discarding pores with an area $<0.5 \mu \mathrm{m}^{2}$, yielding $2.7-$ $3.1 \times 10^{5}$ pores per sample. The mean pore area was calculated averaging the area of all pores detected through the scanned volume; the mean area percentage occupied by the pores was calculated averaging the mean area percentage occupied by the pores for each plane. Density of the mats was measured by measuring sizes using a caliper and micrometer, and an ultrahigh sensitive scale. 


\section{Mechanical characterization of mats}

Mechanical characterization was achieved by applying tensile-strain tests to specimens prepared from the PLA and PLA-SG5 nanofiber mats, using a Zwick-Roell Z0.5TN testing machine (Zwick Roell Group, Ulm, Germany) at RT. Conditions include a strain rate of $10 \mathrm{~mm} / \mathrm{min}$. Specimens of $11.5 \mathrm{~cm}$ in nominal length and $2 \mathrm{~cm}$ in width were created using a stainless steel punch. The tensile stress of each membrane was calculated on the nominal cross-sectional area of the tensile specimens. Young's modulus (linear region before $0.2 \%$ plastic strain) and tensile strength (zero slope) were calculated from each corresponding tensile-strain curve. The width and thickness of each sample were measured using a caliper and a digital micrometer. Average thickness of the test specimens ranged from 0.1 to $0.15 \mathrm{~mm}$.

\section{Calcium release}

Given the importance that calcium ions play regarding cellular activity, dissolution tests were carried out to monitor the release profile of the calcium contained in the PLA-SG5 mats during their degradation. Calcium release measurement from the mats was performed in cell culture medium at different time points. Mats were cut in squares of $1.7 \times 1.7 \mathrm{~cm}$, sterilized under UV for 15 min on each site, and introduced separately in a 24 -well plate. Then, $500 \mu \mathrm{L}$ of medium, consisting of calcium-free Dulbecco's modified Eagle's medium (DMEM) (Gibco), $2 \mathrm{~mm}$ L-Glutamine (Invitrogen), $100 \mathrm{U} / \mathrm{mL}$ penicillin/streptomycin $(\mathrm{P} / \mathrm{S})$ (Gibco), and 10\% (v/v) fetal bovine serum (FBS) (Sigma-Aldrich), was added in the wells and samples were incubated at $37^{\circ} \mathrm{C}$ in $5 \%(\mathrm{v} / \mathrm{v}) \mathrm{CO}_{2}$. At each time point (30, 60, 90, $120 \mathrm{~min}$, and 24, 48, and $72 \mathrm{~h}) 100 \mu \mathrm{L}$ was removed to be analyzed and replaced with fresh medium. Calcium concentration was assessed with the colorimetric method 0Cresolphthalein Complexone $\left(\right.$ Sigma ${ }^{26}$ reading absorbance at $570 \mathrm{~nm}$ on the Infinite M200pro microplate reader (Tecan). Cumulative calcium release was calculated taking into account the total calcium moles cumulated at each time point, including the ones removed over time and removing the background signal from the medium. $\mathrm{pH}$ was measured with a Laquatwin pHmeter (B-712; Horiba). Five replicates $(n=5)$ were used per condition.

\section{In vitro evaluation of PLA-SG5 biocompatibility}

Evaluation of viability of cells cultured for $24 \mathrm{~h}$ in the medium previously incubated with the fibers was assessed with the alamarBlue ${ }^{\mathrm{TM}}$ (Thermo Scientific) reagent following the manufacturer's instructions. For this experiment, commercially available human dermal fibroblasts from healthy adults (PromoCell) were used. These cells were expanded in a complete culture medium (CCM) consisting of DMEM, $10 \% \mathrm{FBS}, 2 \mathrm{mM}$ L-Glutamine, and $100 \mathrm{U} / \mathrm{mL} \mathrm{P} / \mathrm{S}$ at $37^{\circ} \mathrm{C}$ in $5 \%(\mathrm{v} / \mathrm{v}) \mathrm{CO}_{2}$ changing medium every 3 days and they were used at passages $4-7$.

Conditioned medium was prepared by adding $500 \mu \mathrm{L}$ of CCM on previously UV-sterilized mats of $1.7 \times 1.7 \mathrm{~cm}$ and incubating them for $24 \mathrm{~h}$ at $37^{\circ} \mathrm{C}$ in a $\mathrm{CO}_{2}$ incubator. Medium incubated with mats from the same condition, either PLA or PLA-SG5 mats, were pooled before adding it to the cells. Cells (15,000 cells/well) were seeded in 24-well plates in CCM and, after $24 \mathrm{~h}$, conditioned medium was added. At 1 and 4 days posttreatment, fluorescence was measured at Ex/Em wavelength of 530/590 nm using a microplate reader (Infinite M200 Pro; Tecan). Eight replicates $(n=8)$ were used per condition, and the experiment was repeated three times. Obtained values were normalized to the average value corresponding to the control sample with nonconditioned medium on day 1.

\section{In vivo wound healing studies}

Animal experiments were performed following a procedure approved by the Institutional Animal Experimentation Ethics Committee (Consejo Superior de Investigaciones Científicas, CSIC and the Generalitat de Catalunya) (Approval No. CEA-OH/ 10727/2). Thirty-two 8-week-old male BKS.Cg$D o c k 7^{m}+/+L e p r^{d b} / \mathrm{J}$ mice $(d b / d b)$ were obtained from Charles River Laboratory. This model was selected because the animal is leptin receptor deficient and represents a type II diabetes model characterized by hyperglycemia, obesity, hyperinsulinemia, and impaired wound healing.

Once received, all mice were housed in specific pathogen-free barrier facility and were acclimatized to their new environment for 7 days before the beginning of the study. Animals were fed on a pellet diet of LabDiet ${ }^{\circledR} 5 \mathrm{~K} 52$ formulation (6\% fat) and water ad libitum.

To generate pressure ulcers on the dorsal side of mice a previously reported model was used that consisted in performing cycles of ischemia/reperfusion (IR) with the external application of two magnets. ${ }^{27}$ On the first day of the experiment, mice were anesthetized in a chamber with $5 \%$ isoflurane and maintained at $2 \%$ isoflurane during the procedure. Mice backs were shaved with an electric 
razor and cleaned with $70 \%$ ethanol. Then, the skin was gently pulled and placed between two round ceramic magnetic plates $(12 \mathrm{~mm}$ diameter, $5 \mathrm{~mm}$ thickness; IMA) previously sterilized with ethanol, pinching the epidermis, dermis, hypodermis, and panniculus carnosus. An unpunched space of 0.5 to $1 \mathrm{~cm}$ was left between magnets so that two separate injuries could be generated on each animal. Six IR cycles were performed in each mouse to generate pressure ulcers. A single IR cycle consisted of a 12-h period of magnet placement, followed by a release or rest period of $12 \mathrm{~h}$. Animals were anesthetized for magnet placement, but not for magnet removal, and magnet sterility was ensured by spraying them with $70 \%$ ethanol and exposing them under UV before each application. After anesthesia, animals were monitored for a few minutes until they returned to normal activity. This process produced two circular ulcers covered by necrotic tissue separated by a bridge of healthy skin.

Before treatment application, mice were anesthetized, and necrotic tissue was mechanically removed. Control mats (Mepilex ${ }^{\circledR}$; Mölnlycke Health Care), PLA mats, and PLA-SG5 mats were applied to each wound. Each animal received two different treatments, one treatment per wound. To maintain mats in site, they were covered with an adhesive Plaster (Coverplast ${ }^{\circledR}$ Latex-free; BSNmedical) and further secured with an elastic gauze (Genové Dermatologics). Mat change was performed every $24 \mathrm{~h}$ placing animals under anesthesia.

\section{Wound closure measurements}

Right before treatment application and at every change, digital imaging of wounds was performed using a digital camera (Mavica FD91; Sony) and the ORCA-2BT Imaging System (Hamamatsu Photonics) provided with a C4742-98-LWG-MOD camera fitted with $512 \times 512$-pixel charge-couple device cooled at $-80^{\circ} \mathrm{C}$. Wound size was quantified from images obtained with the abovementioned camera using the Wasabi image analysis software (Hamamatsu Photonics). The area measured at each time point was normalized to the size of the wound before dressing application (day 0).

\section{Histological examination}

On 3 and 8 days posttreatment, eight animals from each group were sacrificed by cervical dislocation. Then, wounds were excised with margins of $2 \mathrm{~mm}$, cut in half, and fixed in $10 \%$ neutral buffered formalin solution (Sigma-Aldrich) for $24 \mathrm{~h}$. Samples were dehydrated in alcohol and paraffin embedded. Sections $(8 \mu \mathrm{m})$ were stained against Hematoxylin and Eosin (H\&E) and modified Mas- son's Trichrome (Sigma-Aldrich) for examining reepithelialization and collagen deposition, respectively. Low magnification images of the stained tissue were acquired with an Olympus DP72 camera from an Olympus Macro Zoom microscope MVX10 (Olympus Life Science), and for high magnification images, a Nikon E600 microscope with an Olympus DP72 camera was used. ImageJ analysis software was used to quantify the percentage of blue collagen-stained area relative to the total area of the wound bed.

Wound specimens were immunostained for CD31 to evaluate vascular angiogenesis. Sections were deparaffinized, rehydrated using ethanol gradients, and placed in phosphate-buffered saline (PBS). Antigen retrieval was performed by incubating the samples for $20 \mathrm{~min}$ at $95^{\circ} \mathrm{C}$ in a sodium citrate buffer solution containing $10 \mathrm{mM}$ sodium citrate tribasic dihydrate (Sigma-Aldrich) with $0.05 \%$ Tween ${ }^{\circledR} 20$ (Sigma-Aldrich) (pH 6.0) and then endogenous peroxidase was quenched using $3 \%(\mathrm{v} / \mathrm{v})$ hydrogen peroxide for $5 \mathrm{~min}$ at RT. Tissues were blocked with $2 \%$ goat serum, in PBS, for $30 \mathrm{~min}$ at RT. Primary antibody against CD31 (ab28364; Abcam, United Kingdom) was used at 1:35 in $2 \%(\mathrm{w} / \mathrm{v})$ BSA in PBS, and incubated overnight at $4^{\circ} \mathrm{C}$. After three washes with PBS, the biotinylated secondary antibody goat anti-rabbit (ab128978; Abcam) was added and incubated for $1 \mathrm{~h}$ at RT. Samples were subsequently incubated with streptavidin peroxidase (ab128985; Abcam), washed three times, and developed with $3^{\prime}$ diaminobenzidine (DAB) staining solution (ab 64239; Abcam). Counterstaining was performed using Harris Hematoxylin. Samples were then mounted using Eukitt medium (Panreac) and viewed by light microscopy. CD31-positive stained vessels were analyzed in sections of eight different skin samples. The number of vessel per unit area $\left(\mathrm{mm}^{2}\right)$ in six random fields per section was quantified using ImageJ software. ${ }^{25}$

\section{Statistical analysis}

Statistical analysis was performed with Graph Pad Prism 5.0 (GraphPad Software, Inc., San Diego, CA).

The significance level was measured by comparing the data between groups by performing oneway analysis of variance followed by Tukey's test. Significance levels were set at $* p \leq 0.05$. Histological examination was performed by capturing at least six fields per section $(n=8)$ and images were analyzed using ImageJ software. Unless otherwise indicated, all graphical data are reported as mean \pm standard deviation. 
A

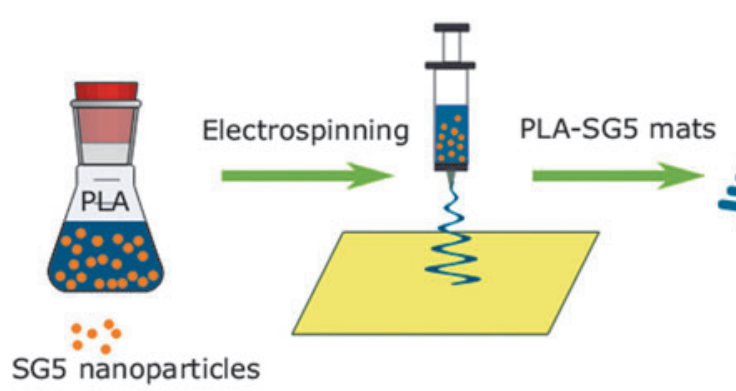

D
In vivo wound healing

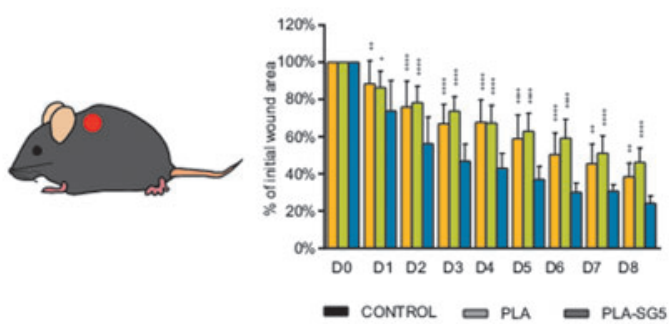

B Morphological characterization
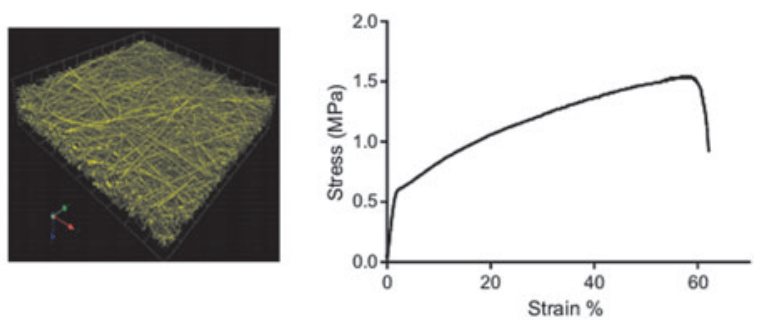

C In vitro biocompatibility
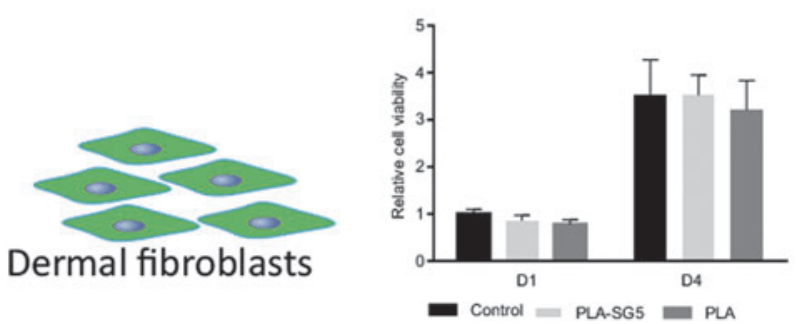

Figure 1. Schematic representation of the workflow. (A) Fabrication of PLA-SG5 mats by electrospinning. (B) Morphological characterization of PLA-SG5 mats by CLMS, FE-SEM, contact angle, and ion release. (C) Biocompatibility of PLA-SG5 using human dermal fibroblasts. (D) In vivo evaluation of PLA-SG5 mats using a pressure ulcer model in diabetic mice. CLMS, confocal laser microscopy scanner; FE-SEM, field emission scanning electron microscopy; PLA, poly(lactic acid).

\section{RESULTS}

\section{Dressing characterization}

Electrospun mats of PLA fibers containing, or not, ormoglass calcium phosphate SG5 particles were synthesized and imaged by SEM. Both PLA and PLA-SG5 mats presented different fiber diameters: $1208.5 \pm 126.2 \mathrm{~nm}$ for pure PLA fibers and

F2 648.1 $\pm 72.2 \mathrm{~nm}$ for PLA-SG5 (Fig. 2A, B). The thickness of the mats used for this study was between 200 and $260 \mu \mathrm{m}$. SG5 particles were observed embedded within the PLA fibers (Fig. 2B). Representative stress and strain typical curves of PLA and particle-loaded PLA mats showed an initial elastic region followed by failure at tensile strength (Fig. 2C). Quantitative analysis on the mechanical properties showed that average approached elastic modulus and tensile strength of the PLA mats were significantly higher than those of the PLA loaded with particles. Approached elastic moduli of PLA-SG5 mats were $31.33 \pm 7.95 \mathrm{MPa}$. These values were much lower than the elastic moduli of the pure PLA mats at $60.08 \pm 2.50 \mathrm{MPa}$. Similarly, tensile strength of PLA-SG5 mats was $1.35 \pm 2.04 \mathrm{MPa}$, which was lower than the PLA mats of $2.05 \pm 0.14 \mathrm{MPa}$.

As can be observed in the recorded videos, contact angle tests show an increased hydrophilicity of PLA-SG5 mats compared with PLA mats
(Supplementary Videos S1 and S2). As can be seen in the recorded video, the water drop is immediately absorbed by capillarity by the PLA-SG5 mats, whereas this effect was not observed when PLA mats were tested (Supplementary Videos S1 and S2).

A gradual release of calcium was measured for the PLA-SG5 mats when incubated in cultureconditioned medium (CCM), reaching its maximum after $48 \mathrm{~h}$ with values between 3 and $4 \mathrm{mM}$ (Fig. 2D). As expected, particle-free PLA fibers did not release calcium. In addition, conditioned medium did not present significant changes in $\mathrm{pH}$ (data not shown).

\section{Porosity}

Figure $3 \mathrm{~A}$ and $\mathrm{B}$ shows reconstructions after image analysis from the CLSM sections.

Pore size distribution analysis indicated pore sizes ranged from 5 to $105 \mu \mathrm{m}^{2}$ in PLA mats and from 5 to $95 \mu \mathrm{m}^{2}$ in PLA-SG5 mats, being most of them around 5 up to $25 \mu \mathrm{m}^{2}$ for both type of mats (Fig. 3C, D). Table 1 shows a small reduction of the total pore surface percentage from $57.3 \pm 4.3$ (pure PLA) to $48.3 \pm 3.9$ (PLA-SG5) and surfaceto-volume ratio remains around 3.5 and 3.4 in both cases. The two conditions, however, differed in their pore density: for pure PLA fibers it was 

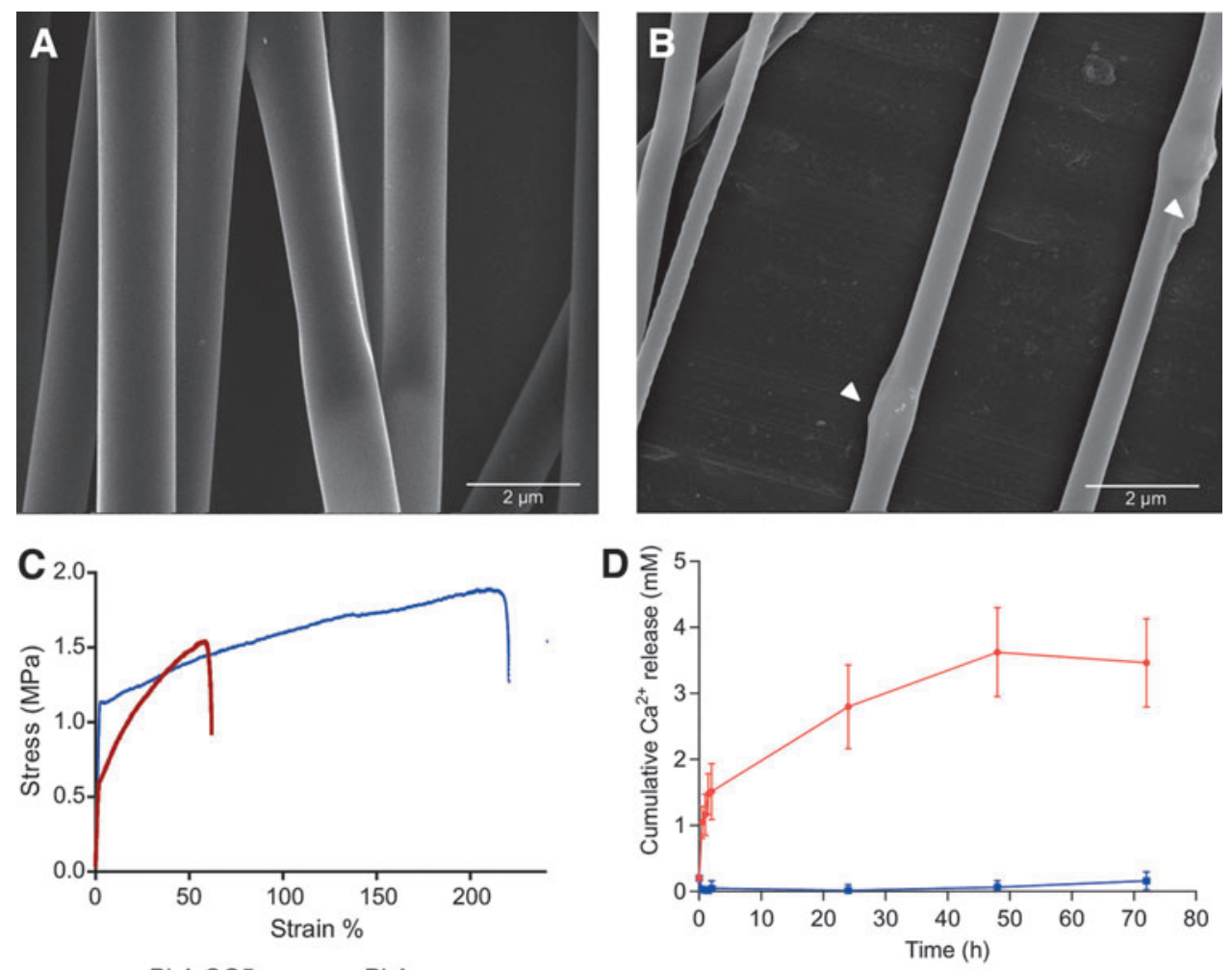

$$
\text { - PLA-SG5 - PLA }
$$$$
\rightarrow \text { PLA-SG5 } \rightarrow \text { PLA }
$$

Figure 2. Characterization of electrospun PLA mats with and without SG5 particles. (A, B) PLA (left) and PLA-SG5 (right) mats imaged with SEM. Arrowheads indicate the presence of particles embedded in the fibers. (C) Representative tensile-stress curves of mats. (D) Cumulative calcium release over time of mats in CCM. ( $n=5$, mean \pm SD). CCM, complete culture medium; SD, standard deviation.

$0.18 \pm 0.04 \mathrm{mg} / \mathrm{mm}^{3}$, whereas for PLA-SG5 fibers it decreased to $0.11 \pm 0.005 \mathrm{mg} / \mathrm{mm}^{3}$. This difference results in significant changes in their specific surface, being $19.3 \pm 0.4$ and $31.0 \pm 1.3 \mathrm{~m}^{2} / \mathrm{g}$, respectively.

\section{Cell viability in vitro}

An indirect viability test on cells exposed to medium conditioned by the release of the PLA and PLA-SG5 mats was performed to detect possible material cytotoxicity. More specifically, adult human dermal fibroblasts were exposed to CCM conditioned for $24 \mathrm{~h}$ with the materials and metabolic activity was measured from alamarBlue reduction after 1 and 4 days posttreatment. Results were compared with a control sample consisting of cells exposed to nonconditioned medium. As shown in

F4 Fig. 4A, no alterations in metabolic activity were detected in dermal fibroblasts exposed to materialconditioned medium.

\section{Wound healing performance and gross examination of the healing process in diabetic mice}

The wound healing efficacy of PLA-SG5 mats in the treatment of chronic wounds was evaluated in vivo. Two pressure ulcers were created on the back of obese and diabetic mice that allowed to compare the healing capability of PLA-SG5 against PLA mats and Mepilex, a commercially available dressing frequently used in the treatment of chronic wounds. Wound area reduction was followed daily for 8 days (Fig. 4B) and values are shown in Fig. 4C. PLA-SG5 treatment presented the fastest healing rate compared with both PLA and Mepilex at every time point analyzed. On the other hand, both PLA and Mepilex showed a comparable percentage of wound closure, since differences were not significant between them.

Gross examination of H\&E-stained sections was also performed (Fig. 5A). The epithelial gap, defined as the distance between the two advancing epithelial tongues, was smaller in the PLA-SG5 group than in the PLA and control groups at 3 and 8 days posttreatment (Fig. 5B). At 8 days, posttreatment differences were also found between PLA and control groups, being the epithelial gap of the PLA group smaller than the control group.

\section{Histopathological analysis of the wound}

We performed the histopathological assessment of control wounds and wounds treated with PLA or 

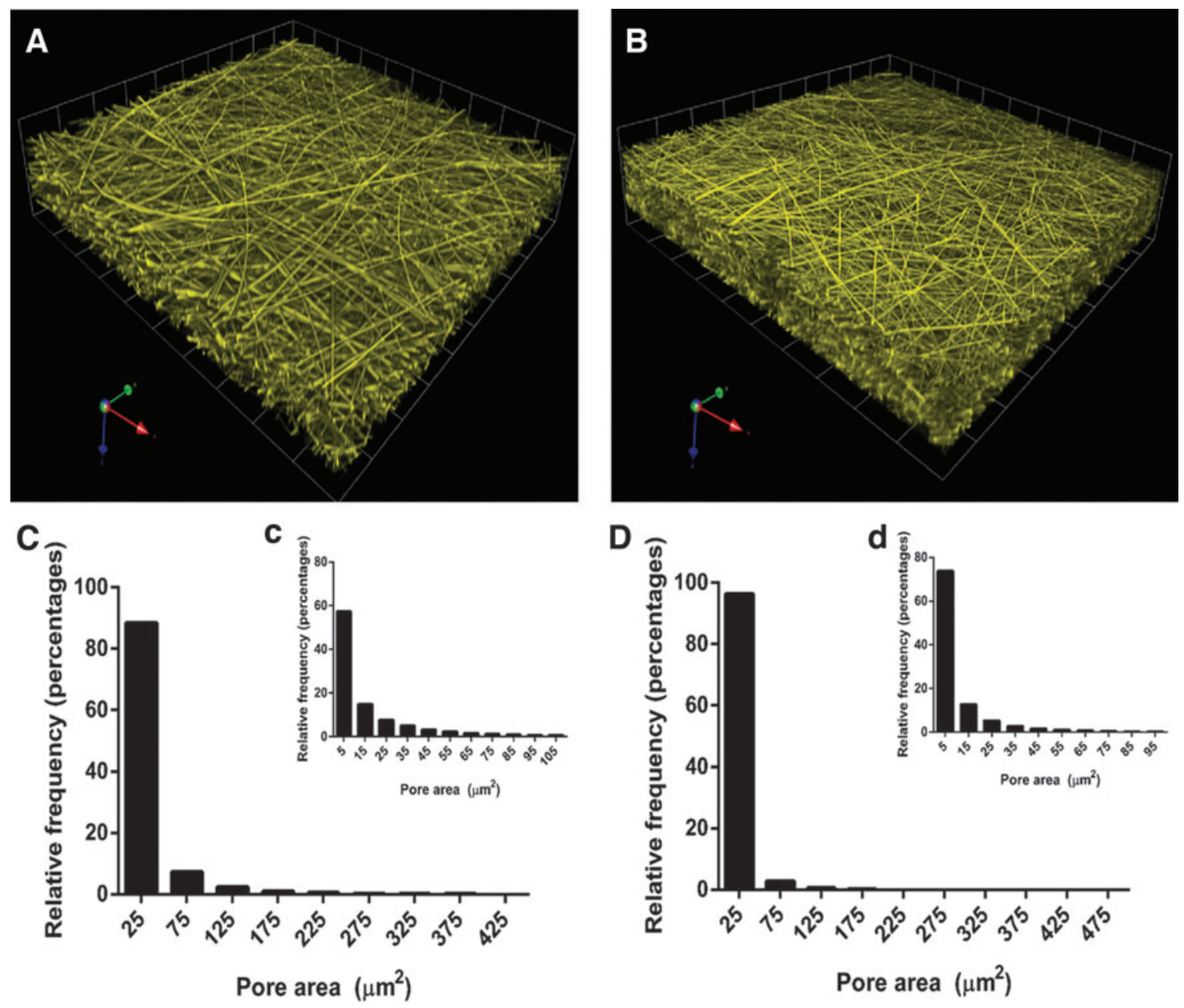

Figure 3. Three-dimensional reconstructions from CLSM sections of (A) PLA and (B) PLA-SG5 mats. Pore size distribution of PLA mats of pore areas (C) 0 $425 \mu \mathrm{m}^{2}$, (c) $0-105 \mu \mathrm{m}^{2}$, and PLA-SG5 mats of pore area (D) 0-475 $\mu \mathrm{m}^{2}$, (d) $0-95 \mu \mathrm{m}^{2}$. CLSM, confocal laser scanning microscopy.

T2 PLA-SG5 mats (Table 2). At day 3 after wounding, none of the treatments affected the number of polymorphonuclear leukocytes. In addition, less fibrin and a higher number of fibroblasts were observed in wounds treated with PLA-SG5. At 8 days postwounding, similar number of inflammatory cells were found between all treatments. Moreover, an increased migration of fibroblasts was observed in PLA-SG5-treated wounds.

\section{Granulation tissue assessment}

H\&E-stained sections were also used to analyze granulation tissue formation at the wound bed. On

Table 1. Bone volume/total volume

\begin{tabular}{lcc}
\hline & PLA & PLA-SG5 \\
\hline \% Surface area occupied by pore & $57.3 \pm 4.3$ & $48.3 \pm 3.9$ \\
Surface $/$ volume $\left(\mu \mathrm{m}^{-1}\right)$ & $3.5 \pm 0.6$ & $3.4 \pm 0.1$ \\
Density $\left(\mathrm{mg} / \mathrm{mm}^{3}\right)$ & $0.18 \pm 0.04$ & $0.11 \pm 0.005$ \\
Specific surface $\left(\mathrm{m}^{2} / \mathrm{g}\right)$ & $19.3 \pm 0.4$ & $31.0 \pm 1.3$ \\
\hline
\end{tabular}

PLA, poly(lactic acid). the third day of treatment, PLA- and PLA-SG5treated wounds showed increased cellularity in the wound area compared with Mepilex (Table 2) (Fig. 6A).

\section{Collagen formation at the wound site}

Since collagen formation is a fundamental component of wound healing, sections were stained (Masson's Trichrome) to assess collagen deposition at the wound bed (Fig. 6B). Regarding collagen distribution, an increased collagen deposition was observed in wounds treated with PLA-SG5 mats at 3 and 8 days postwounding than in wounds treated with the commercial dressing or PLA mats (Fig. 6C).

\section{Vessel quantification}

Neovascularization is a key process for the successful healing of wounds and, for this reason, vessel density was analyzed from sections immunolabeled against CD31, a marker for endothelial cells (Fig. 7A, B). Sections from wounds 


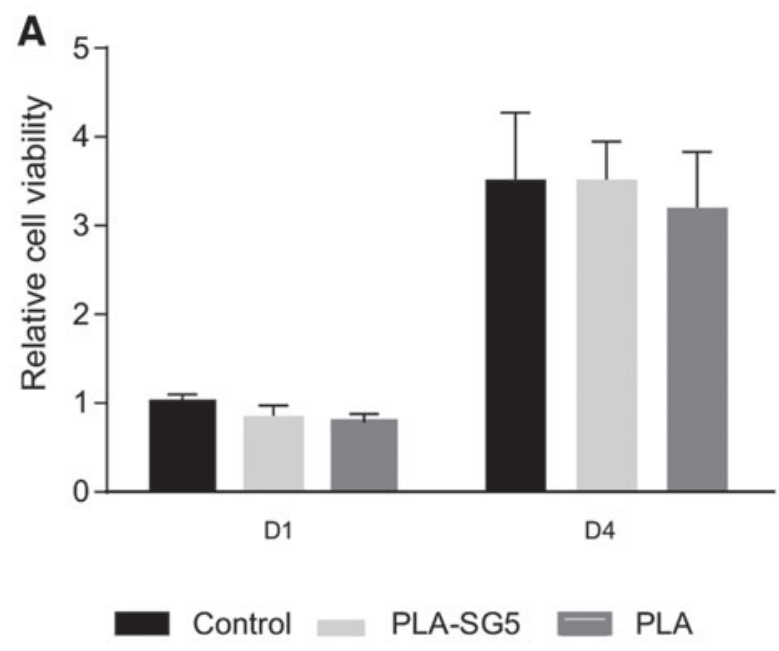

B

D0

D3

D8
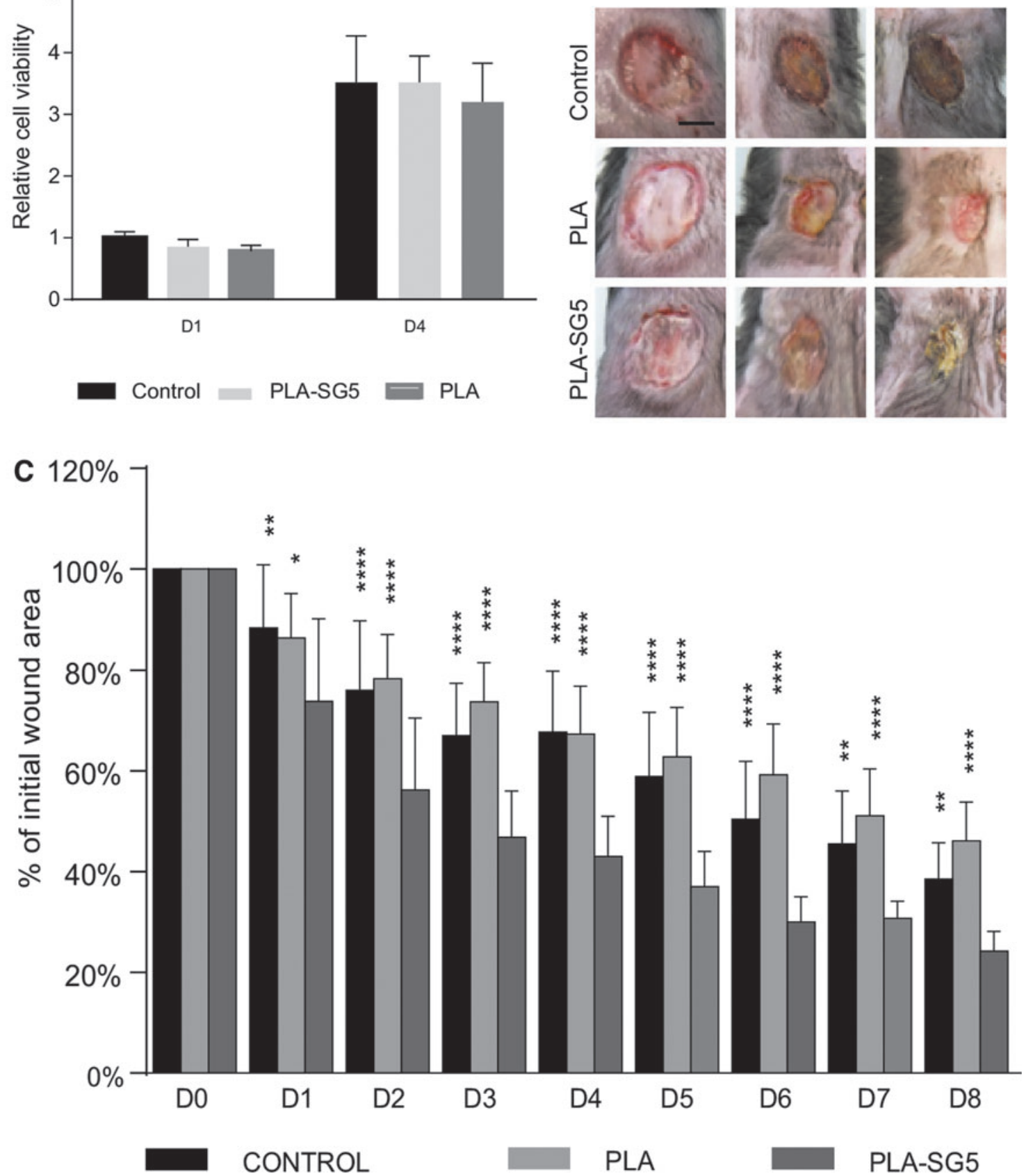

Figure 4. Assessment of human dermal fibroblasts viability in conditioned medium. (A) Adult human dermal fibroblast from healthy donors were exposed to CCM previously incubated with PLA or PLA-SG5 mats, and metabolic activity was quantified after 1 and 4 days posttreatment. Values were normalized to the average of the control sample exposed to nonconditioned medium on day 1. $(n=8$, mean \pm SD). Assessment of wound healing size at different time points. (B) Representative images of the group treated with Mepilex ${ }^{\circledR}$, PLA, and PLA-SG5 on day 0,3 , and 8 posttreatment. (C) Percentage of wound size relative to the initial size during the course of the experiment. Data are expressed as the mean $\pm S D(n=8) .{ }^{*} p<0.05$ (vs. PLA-SG5), ${ }^{* *} p<0.01$ (vs. PLA-SG5), ${ }^{* * *} p<0.0001$ (vs. PLA-SG5). Scale bar $=0.5 \mathrm{~mm}$.

treated with PLA-SG5 for 3 days showed significantly higher vessel density than both treatment controls (Fig. 7B). On day 8 posttreatment, vessel density from the PLA-SG5 group regressed and reached similar values to PLA and Mepilex treatments (Fig. 7B).

\section{DISCUSSION}

Chronic wounds are a socioeconomic burden for first-world countries and new therapies are needed to accelerate their healing. Biological-based therapies have been developed over the last few years with promising results, but due to regula- 

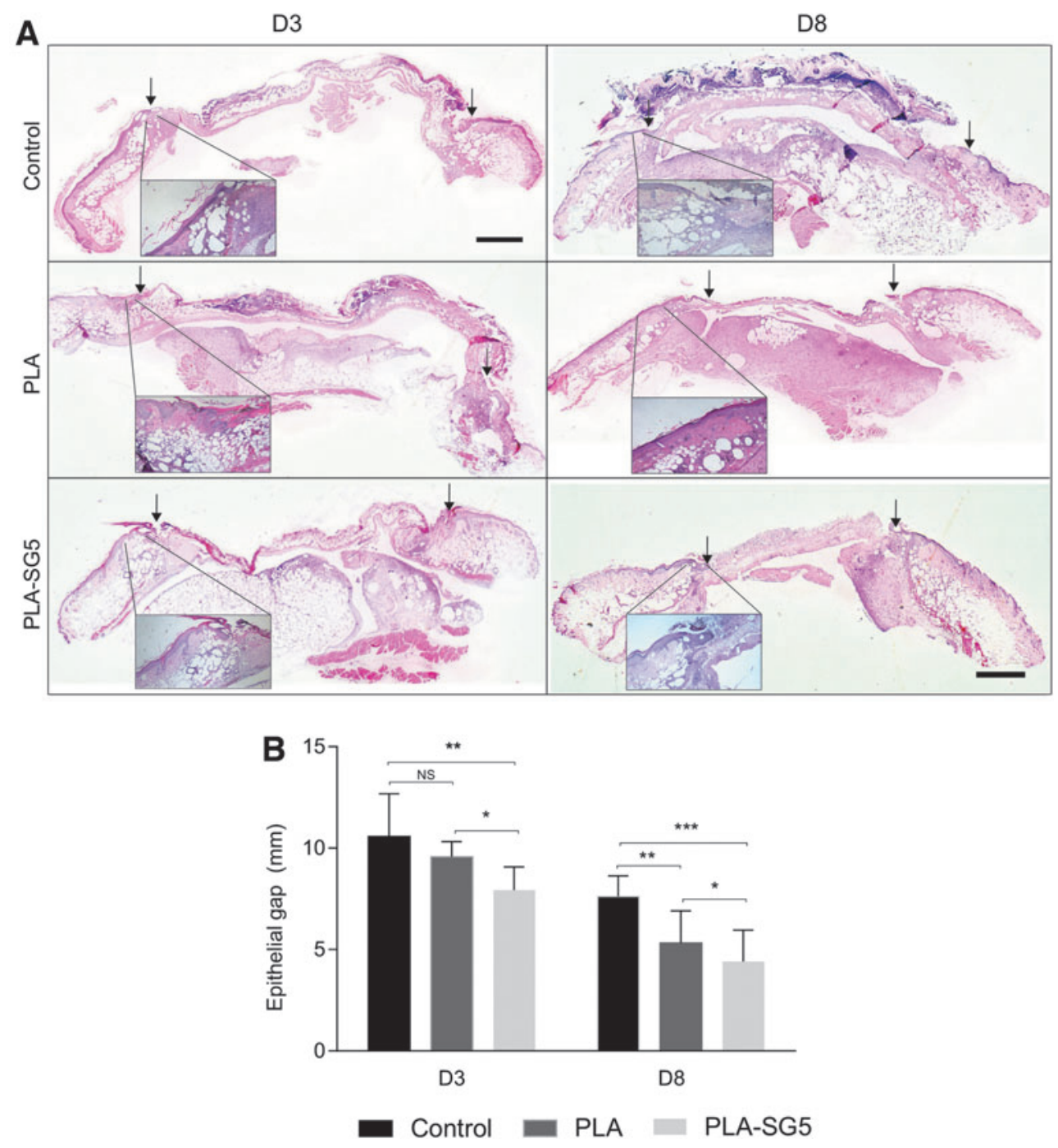

Figure 5. Analysis of wound structure from sections stained with H\&E. (A) Representative images of wound sections stained with H\&E from day 3 and 8 posttreatment. Arrows delimit the unepithelialized surface of the wound. (B) Quantification of the epithelial gap length from each experimental condition on day 3 and 8 posttreatment. Data are expressed as the mean \pm SD of at least five wounds from different animals. $+p<0.05,{ }^{* *} p<0.01$ (vs. PLA-SG5). ${ }^{* * *} p<0.001$ (vs. PLA-SG5). Scale bar $=1 \mathrm{~mm}$.

\begin{tabular}{|c|c|c|c|c|c|c|}
\hline & $\begin{array}{l}\text { Day } 3 \\
\text { Control }\end{array}$ & $P L A$ & PLA-SG5 & $\begin{array}{l}\text { Day } 8 \\
\text { Control }\end{array}$ & PLA & PLA-SG5 \\
\hline \multicolumn{7}{|l|}{ Inflammation status } \\
\hline PMN & +++ & +++ & +++ & ++ & ++ & ++ \\
\hline Lymphocytes & ++ & ++ & +++ & + & ++ & +++ \\
\hline Plasma cells & + & + & + & + & + & + \\
\hline Fibrin & ++ & ++ & + & + & + & + \\
\hline \multicolumn{7}{|l|}{ Repair } \\
\hline Fibroblasts & ++ & ++ & +++ & + & ++ & +++ \\
\hline \multicolumn{7}{|l|}{ Fibrous tissue } \\
\hline Loose & ++ & ++ & ++ & ++ & ++ & ++ \\
\hline Dense/scar & - & - & - & - & - & - \\
\hline Granulation tissue & + & ++ & +++ & + & ++ & ++ \\
\hline
\end{tabular}

PMN, polymorphonuclear leukocytes. tory, economic, and safety concerns their translation into the clinics has been very limited. Owing to the wound healing capability recently reported by bioceramics, together with their lower cost of production and smaller safety risks, these materials arise like an interesting alternative to produce new translational off-the-shelf dressings. In this study, we tested the healing capability of electrospun PLA mats incorporating the calcium phosphate ormoglass particles SG5 on a pressure ulcer model in diabetic mice. The presence of the particles stimulated a faster wound area reduction, increased vascularization, collagen deposition, and formation of granulation tissue. In addition, the generated wound presented increased reepithelialization and improved morphogenesis 

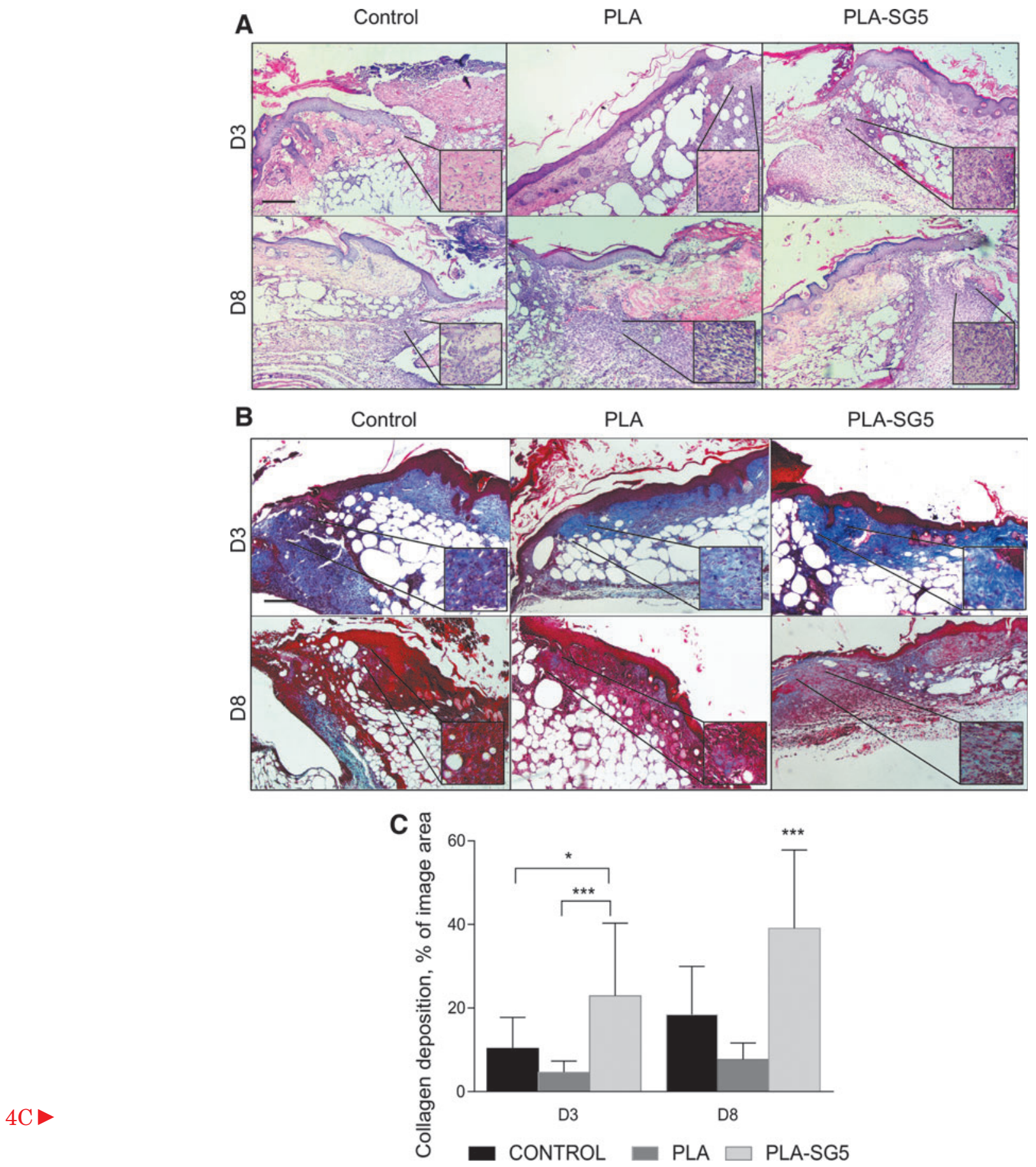

Figure 6. Analysis of granulation tissue and collagen deposition from sections stained with (A) H\&E and (B) Masson's Trichrome. Representative images of all conditions from wound sections stained with H\&E from day 3 and 8 posttreatment at two different magnifications showing the granulation tissue. (C) Increased collagen deposition in PLA-SG5 treated wounds at 3 and 8 days postwounding $\mathrm{S}$ bar $=250 \mu \mathrm{m}$.

compared wit a commercially available dressing frequently used in the clinics.

After generating the PLA-SG5 composite using electrospinning, its structure and biocompatibility were evaluated. Our results indicate a slight decrease in mechanical properties of the PLA-SG5 mats compared with PLA mats.
This could be explained by a reduction of diameter fiber in the PLA-SG5 mats.

Mats were composed by a dense network of PLAbased fibers with diameters smaller than a micron. In this work, porosity of the mats was assessed using CLMS. This is an interesting technique since it allows obtaining multiple stacks of layers that 
A
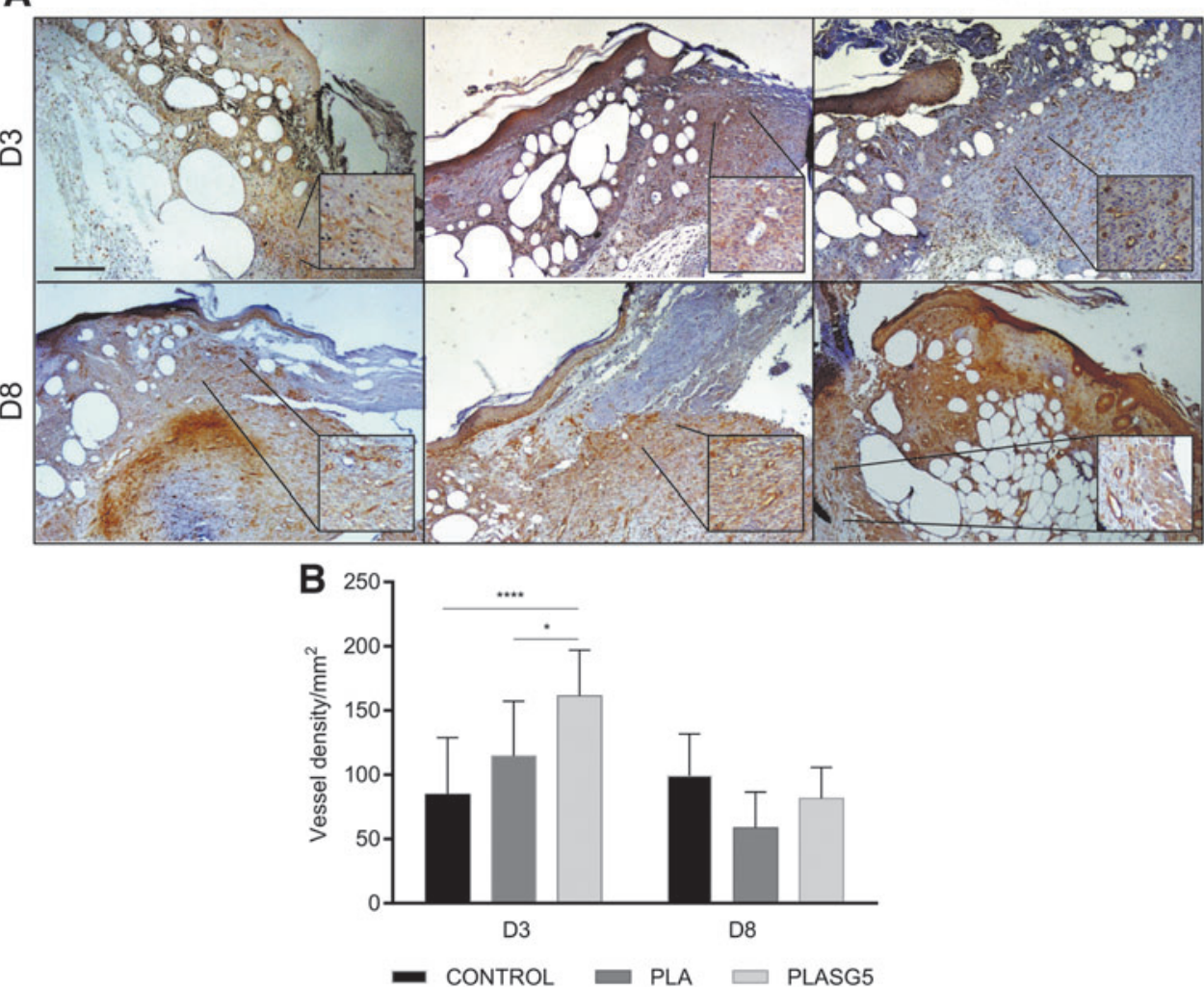

Figure 7. Imaging and quantification of blood vessels immunolabeled for CD31. (A) Representative images of immunostaining against CD31 of sections of wounds treated with Mepilex (Control), PLA, and PLA-SG5 mats at 3 and 8 days postwounding. (B) Quantification of the number of vessels from the immunostained images. ${ }^{*} p<0.05$ (vs. PLA-SG5). ${ }^{* * *} p<0.0001$ (vs. PLA-SG5). Scale bar $=250 \mu \mathrm{m}$.

can be easily analyzed with imageJ software. The impossibility to use other techniques more aggressive with polymeric nanostructured materials, such as Brunauer, Emmett, Teller, or mercury porosimetry, makes this approach a suitable strategy to assess this characterization. The differences in porosity detected between the PLA and the PLA-SG5 mats are probably related to the extra volume resulting from the addition of nanoparticles. The higher porosity and hydrophilicity found in PLASG5 mats may allow a good contact of the fibers with the physiological fluid of the wound. Actually, the surface-to-volume ratio in both cases is about 3.43.5 , indicating that nanofibers provide a high specific surface confirming a homogeneous degradation and a lack of diffusion-controlled processes. In fact, the addition of SG nanoparticles increases the specific surface to almost twofold (Table 1), probably due to the decrease in fiber thickness by half. Possible reasons for this reduction can be multiple, but it seems that the remaining low amounts of alcohol present in the ormoglasses can decrease viscosity of the slurry. All in all, this makes the scaffold faster in terms of degradation although mechanical properties are affected.
Both dressings have mechanical properties that facilitate its application and removal and maintain its flexibility during the healing process. However, the higher specific surface, porosity, and hydrophilicity of the PLA-SG mats allow a good interaction of the dressing with the wound fluids. These features are important for absorption of wound exudates and maintenance of a moist environment at the wound site.

PLA-SG5 mats presented a gradual calcium release for up to $48 \mathrm{~h}$, reaching values between 3 and $4 \mathrm{mM}$ without significantly altering the $\mathrm{pH}$ of the medium. Most of the release happened along the first $24 \mathrm{~h}$. As reported in our previous study, SG5 nanoparticles release not only calcium but also phosphorous $(\mathrm{P})$ in the $\mathrm{mM}$ range and Titanium (Ti), was only partially liberated within the $\mu \mathrm{M}$ rate. ${ }^{18}$ In addition, medium conditioned by this material for $24 \mathrm{~h}$ did not alter metabolic activity of human dermal fibroblast over time when tested in vitro.

The choice of the animal wound healing model is of great relevance when testing a therapy because the healing response can vary significantly depending on the type of wound and the presence of 
complication factors-for example, advanced age, obesity, diabetes, etc. ${ }^{28}$ In our study, the in vivo model chosen to test the healing capability of the newly synthesized mats consisted of pressure ulcer wounds generated in mice. Moreover, the animals used presented common comorbidities associated with chronic wounds that complicate the healing process: diabetes and obesity. While diabetes is an acknowledged pressure ulcers' risk factor ${ }^{29}$ and obesity is a comorbidity often present in diabetic patients that can further complicate the healing process. ${ }^{30}$

Although mice and human present different healing mechanisms (contraction in mice vs. reepithelialization in humans) ${ }^{31}$ this model enabled us to test our developed dressing on a wound with a much more similar pathophysiology to human chronic wounds than previously reported models. Studies that have evaluated the healing capability of bioceramics have generally used full-thickness wounds generated with scissors on healthy mice or rats ${ }^{32}$ which does not model correctly the pathophysiology of chronic wounds. Only a few studies used diabetic animals, ${ }^{33,34}$ presenting impaired wound healing capability, but, again, the type of wound generated was an acute fullthickness injury. In the present study, by applying several ischemia/reperfusion cycles, we created a full-thickness wound with necrosis damage down to the subcutaneous tissue and decreased blood flow to the injured area. ${ }^{27}$ The control used consisted of either particle-free PLA mats or a portion of Mepilex, which is a frequently used dressing for the treatment of low-exuding chronic wounds. While the PLA control enabled us to discern the specific healing contribution of the calciumreleasing particles, the Mepilex control allowed to compare the efficacy of the novel treatment to a market product.

Quantification of the wound area revealed a significant reduction in wounds treated with PLA-SG5 mats compared with both controls, from the first day of treatment to the end of the study. On the other hand, the epithelial gap measured in the PLA-SG5 wound-treated sections displayed differences against the PLA mats and Mepilex control.

During the remodeling and repair phase of wound healing, degradation and deposition of new extracellular matrix by fibroblasts take place. Yet, in chronic wounds the synthesis of new tissue is prevented by several factors. ${ }^{35}$ Treatment of wounds with PLA-SG5 mats resulted in an increased collagen synthesis compared with the other treatments. The increased collagen deposi- tion correlates with the higher number of fibroblasts observed in the PLA-SG5 treated wounds. These in vivo observations are in line with our previous study using dermal fibroblasts that reported an increased production of collagen when these cells were treated with both the ionic dissolution of SG5 nanoparticles and extracellular calcium. ${ }^{18}$ Altogether, these results indicate that PLA-SG5 mats stimulate fibroblasts' migration and de novo deposition of collagen fibers contributing to an improved wound healing in chronic wounds, in which the expression of collagen by fibroblasts is inhibited. ${ }^{36}$

Next, we hypothesized that the accelerated healing promoted by PLA-SG5 could be triggered by an increase in vascularization, since bioceramics have been reported to stimulate angiogenesis not only in vitro, ${ }^{37,38}$ but also in wound healing studies in vivo. ${ }^{34,39}$ Indeed, as early as 3 days posttreatment, vessel density was significantly increased in the PLA-SG5-treated wounds compared with the controls. In addition, these wounds also presented increased cellularity at the wound bed. These two features seem to indicate the presence of more granulation tissue in PLA-SG5treated wounds. On day 8, however, vessel density levels for the PLA-SG5 condition decreased and were similar to the PLA and Mepilex treatments. At this time point, cell density in the injured site was also similar among conditions.

These decrease in vessel density is a normal process that occurs during wound healing. After the growth of blood vessels into the wounds, most of the newly formed vessels regress until eventually the density of blood vessels returns to that of normal, uninjured skin. ${ }^{40,41}$ Thus, increased angiogenesis followed by a regression in vessel density is desired by chronic wound healing therapies. The controlled blood vessel formation observed by applying PLA-SG5 mats is an important clinical consideration when compared with growth factorbased therapies that may led to an uncontrolled angiogenesis resulting in an increased risk of malignancy in patients. ${ }^{42}$

As mentioned earlier, diabetic wounds impacted by insufficient angiogenesis show decreased vascularity. ${ }^{43}$ One of the reasons for the impaired angiogenesis is the low levels of proangiogenic factors present in chronic wounds. ${ }^{44,45} \mathrm{In}$ previous studies we have demonstrated that calcium-releasing particles stimulate the release of angiogenic growth factors in endothelial progenitor cells ${ }^{46}$ and mesenchymal stromal cells. ${ }^{47}$ Hence, it is possible that the improved wound healing effects observed by the SG5-mats might be 
attributable, in part, to a stimulation of angiogenic factor production that resulted in increased angiogenesis. In chronic wounds, the formation of new blood vessels is limited by several factors, including diabetes and obesity. Despite the fact that these comorbidities were inherent in our in vivo model, the presence of SG5 nanoparticles was able to stimulate blood vessel formation in a pressure ulcer wound with impaired vascularization. Based on the findings of the in vivo study presented here, our dressing seems to be a feasible and promising product to reach the market. In addition, SG5 particles are available by cost-effective synthesis with a defined composition, offering an additional advantage in potential clinical application. The PLA-SG5 mat not only accelerate wound closure, which is the main factor prioritized by clinicians when choosing a dressing, but also stimulated granulation tissue formation, one of the main uncovered wound aspects of current dressings. ${ }^{48}$

\section{INNOVATION}

This study evaluated for the first time the healing capability of calcium-releasing bioceramic nanoparticles on a pressure ulcer model with impaired vascularization.

The particles, applied in a dressing composed of PLA nanofibers, accelerated wound closure, promoted increased vascularization, granulation tissue formation, and collagen deposition. The overall performance of the newly designed mat was remarkably better than a commercially available dressing frequently used for the treatment of lowexuding chronic wounds. ${ }^{49}$

As both PLA and calcium phosphate materials are currently accepted for clinical application, this novel off-the-shelf dressing has great potential to reach the market within reasonable time.

\section{ACKNOWLEDGMENTS AND FUNDING SOURCES}

This work was supported by the Spanish Ministry of Economy and Competitiveness (MINECO/ FEDER) through the projects MAT2012-38793 and MAT2015-68906-R, the EuroNanoMed3 project nAngioDerm funded through the Spanish Ministry of Science and Innovation (ref. PCI2019-103648), the Spanish Ministry of Education, Culture, and Sports with the FPU grant (ref. AP-2012-5310), EIT Health (project EIT PoC-2016-SPAIN-03), La
Caixa Banking Foundation through their CaixaImpulse Program and Caixaimpulse 2.0 Consolidate Program (Ref. LCF/TR/CN18/52210003). The authors acknowledge CERCA Program/ Generalitat de Catalunya. O. Castano acknowledges the support from the Serra Hunter program. The authors acknowledge Priscila Giavedoni from the Dermatology Department, Hospital Clinic de Barcelona for the histopathological assessment.

\section{AUTHOR DISCLOSURE AND GHOST WRITING}

No competing financial interests exist. The content of this article was expressly written by the authors listed. No ghostwriters were used to write this article.

\section{ABOUT THE AUTHORS}

Soledad Perez-Amodio received her $\mathrm{PhD}$ in Cellular and Molecular Biology from the University of Amsterdam. She is a Senior Researcher affiliated to the CIBER-BBN in the group of Biomaterials for Regenerative Therapies (IBEC). She is an adjunct professor at the Polytechnic University of Catalonia (UPC). Nuria Rubio, PhD, works at the Cell Therapy Group, Institute for Advanced Chemistry of Catalonia. Olaia F Vila, PhD, works as a Scientist at Gladstone Institutes in San Francisco. Claudia Navarro-Requena, PhD, works as a Researcher and Project Manager at LEITAT Technological Center. Oscar Castano, $\mathbf{P h D}$, is an associate professor at the Electronics and Biomedical Engineering, University of Barcelona. Aitor Sanchez-Ferrero, PhD, works as EU-ITN Project Manager at the Free University of Brussels. Joan Marti-Munoz, PhD, is a postdoctoral fellow at the Center for Research in Medical Devices, University of Ireland Galway. Mercè Alsina-Gibert is Dermatologist at the Clinical Hospital of Barcelona. 
Jeronimo Blanco, PhD, works at the Cell Therapy Group, Institute for Advanced Chemistry of Catalonia. Elisabeth Engel got her PhD in bone metabolism diseases at the Medical School of Autonomous University of Barcelona. She is the principal investigator of the Biomaterials for Regenerative Therapies group at IBEC, and full professor in the Technical University of Catalonia (UPC).

\section{SUPPLEMENTARY MATERIAL}

Supplementary Video S1

Supplementary Video S2

\section{REFERENCES}

1. Guariguata L, Whiting DR, Hambleton I, Beagley J. Global estimates of diabetes prevalence for 2013 and projections for 2035. Diabetes Res Clin Pract 2013;103:137-149.

2. Rodrigues BT, Vangaveti VN, Malabu UH. Prevalence and risk factors for diabetic lower limb amputation: a clinic-based case control study. J Diabetes Res 2016;2016:5941957.

3. Castaño 0, Pérez-Amodio S, Navarro C, Mateostimoneda Á, Engel E. Instructive microenvironments in skin wound healing: biomaterials as signal releasing platforms. Adv Drug Deliv Rev 2018:129:95-117

4. Pang C, Ibrahim A, Bulstrode NW, Ferretti P. An overview of the therapeutic potential of regenerative medicine in cutaneous wound healing. Int Wound J 2017:143:450-459.

5. Frykberg RG, Banks J. Challenges in the treatment of chronic wounds. Adv. Wound Care 2015;4:560582.

6. Miguez-Pacheco V, Hench LL, Boccaccini AR. Bioactive glasses beyond bone and teeth. Acta Biomater 2014:13:1-5.

7. Yu H, Peng J, Xu Y, Chang J, Li H. 1 Bioglass activated skin tissue engineering constructs for wound healing. ACS Appl Mater Interfaces 2016; 8:703-715

8. Ma $W$, Yang $X$, Liang $M$, et al. Fabrication of bioactive glass-introduced nanofibrous membranes with multifunctions for potential wound dressing. RSC Adv 2014:4:60114-60122.

9. Oda Y, Tu C, Menendez A, Nguyen T, Bikle DD. Vitamin $D$ and calcium regulation of epidermal wound healing. J Steroid Biochem Mol Biol 2016; 164:379-385.

10. Day RM, Boccaccini AR, Shurey S, et al. Assessment of polyglycolic acid mesh and bioactive glass for soft-tissue engineering scaffolds. Biomaterials 2004;25:5857-5866

11. Day RM. Bioactive glass stimulates the secretion of angiogenic growth factors and angiogenesis in vitro. Tissue Eng 2005;11:768-777.

12. Li H, He J, Yu H, Green CR, Chang J. Bioglass promotes wound healing by affecting gap junction connexin 43 mediated endothelial cell behavior. Biomaterials 2016:84:64-75.
13. Magee I, Lytton NA, Watt FM. Calcium-induced changes in cytoskeleton and motility of cultured human keratinocytes. Exp Cell Res 1987;172:43-53.

14. Kulesz-Martin MF, Fabian D, Bertram JS. Differential calcium requirements for growth of mouse skin epithelial and fibroblast cells. Cell Tissue Kinet 1984;17:525-533

15. Rokosova B, Bentley JP. Effect of calcium on cell proliferation and extracellular synthesis in arterial smooth muscle cells and dermal fibroblasts. Exp Mol Pathol 1986;44:307-317

16. Wang T, Gu O, Zhao J, et al. Calcium alginate enhances wound healing by up-regulating the ratio of collagen types $\mathrm{I} / \mathrm{III}$ in diabetic rats. Int $\mathrm{J}$ Clin Exp Pathol 2015:8:6636-6645.

17. Dulbecco R, Elkington J. Induction of growth in resting fibroblastic cell cultures by $\mathrm{Ca}++$. Proc Natl Acad Sci U S A 1975;72:1584-1588.

18. Navarro-Requena C, Perez-Amodio S, Castaño O, Engel $E$. Wound healing-promoting effects stimulated by extracellular calcium and calciumreleasing nanoparticles on dermal fibroblasts. Nanotechnology 2018;28:395102.

19. Aguirre A, González A, Planell JA, Engel E. Extracellular calcium modulates in vitro bone marrowderived Flk-1 + CD34 + progenitor cell chemotaxis and differentiation through a calcium-sensing receptor. Biochem Biophys Res Commun 2010;393:156-161.

20. Oliveira H, Catros S, Boiziau C, et al. The proangiogenic potential of a novel calcium releasing biomaterial: impact on cell recruitment. Acta Biomater 2016:29:435-445

21. Martin A, Komada MR, Sane DC. Abnormal angiogenesis in diabetes mellitus. Med Res Rev 2003:23:117-145.

22. Santoro M, Shah SR, Walker JL, Mikos AG. Poly(lactic acid) nanofibrous scaffolds for tissue engineering. Adv Drug Deliv Rev 2016;107:206-212.

23. Sachot N, Castano O, Oliveira $H$, et al. A novel hybrid nanofibrous strategy to target progenitor cells for cost-effective in situ angiogenesis. J Mater Chem B 2016;4:6967-6978

24. Kamudzandu M, Roach P, Fricker R, Yang Y. Nanofibrous scaffolds supporting optimal central nervous system regeneration: an evidence-based review. J. Neurorestoratology 2015:3:123-131.
25. Schindelin J, Arganda-Carreras I, Frise E, et al. Fiji-an Open Source platform for biologicalimage analysis. Nat Methods 2012;9:676-682.

26. Cohen SA, Sideman L. Modification of the o-cresoiphthalein complexone method for determining calcium. Clin Chem 1979;25:1519-1520.

27. Wassermann E, van Griensven M, Gstaltner K, Oehlinger W, Schrei K, Redl H. A chronic pressure ulcer model in the nude mouse. Wound Repair Regen 2009:17:480-484

28. Xue SN, Lei J, Yang C, Lin DZ, Yan L. The biological behaviors of rat dermal fibroblasts can be inhibited by high levels of MMP9. Exp Diabetes Res 2012;12:494579.

29. Lyder CH, Ayello E. Pressure ulcers: a patient safety issue. In: Hughes RG, ed. Patient Safety and Quality: An Evidence-Based Handbook for Nurses. Rockville, MD: Agency for Healthcare Research and Quality (US), 2008:268-299.

30. Anderson K, Hamm RL. Factors that impair wound healing. J Am Coll Clin Wound Spec 2014;4:84-91.

31. Dorsett-Martin W. Rat models of skin wound healing: a review. Wound Repair Regen 2004;12: 591-599.

32. Jebahi S, Oudadesse H, Jardak N, et al. Biological therapy of strontium-substituted bioglass for soft tissue wound-healing: responses to oxidative stress in ovariectomised rats. Ann Pharm Fr 2013; 71:234-242.

33. Mao C, Lin C, Chen X. Enhanced healing of fullthickness diabetic wounds using bioactive glass and Yunnan Baiyao ointments. J Wuhan Univ Technol Mater Sci Ed 2014;29:1063-1070.

34. Lin C, Mao C, Zhang J, Li Y. Healing effect of bioactive glass ointment on full-thickness skin wounds. Biomed Mater 2012;7:045017.

35. Medina A, Scott PG, Ghahary A, Tredget EE. Pathophysiology of chronic nonhealing wounds. J Burn Care Rehabil 2005;26:306-319.

36. Hansen SL, Young DM, Boudreau NJ. HoxD3 expression and collagen synthesis in diabetic fibroblasts. Wound Repair Regen 2003;11:474-480.

37. Leu A, Leach JK. Proangiogenic potential of a collagen/bioactive glass substrate. Pharm Res 2008:25:1222-1229. 
38. Zeng Q, Han Y, Li, H, Chang J. Bioglass/alginate composite hydrogel beads as cell carriers for bone regeneration. J Biomed Mater Res B Appl Biomater 2014;102:42-51.

39. Xu H, Lv F, Zhang Y, et al. Hierarchically micropatterned nanofibrous scaffolds with a nanosized bio-glass surface for accelerating wound healing. Nanoscale 2015;7:18446-18452.

40. Brown NJ, Smyth EAE, Cross SS. Angiogenesis induction and regression in human surgical wounds. Wound Repair Regen 2002;10:245-251.

41. Johnson KE, Wilgus TA. Vascular endothelial growth factor and angiogenesis in the regulation of cutaneous wound repair. Adv Wound Care 2014;3:647-661.

42. Barrientos S, Brem H, Stojadinovic O, Tomic-Canic M. Clinical application of growth factors and cytokines in wound healing. Wound Repair Regen 2014;22:569-578

43. Dinh T, Veves A. Microcirculation of the diabetic foot. Curr Pharm Des 2005;11:2301-2309.
44. Okonkwo UA, Dipietro LA. Diabetes and wound angiogenesis. Int J Mol Sci 2017:18:1-15.

45. Lauer G, Sollberg S, Cole M, et al. Expression and proteolysis of vascular endothelial growth factor is increased in chronic wounds. J Invest Dermatol 2000;115:12-18.

46. Aguirre A, González A, Navarro M, et al. Control of microenvironmental cues with a smart biomaterial composite promotes endothelial progenitor cell angiogenesis. Eur Cells Mater 2012;24:90-106.

47. Navarro-Requena C, Weaver JD, Clark A, et al. Acta Biomaterialia PEG hydrogel containing calcium-releasing particles and mesenchymal stromal cells promote vessel maturation. Acta Biomater 2018;67:53-65.

48. Wu S, Applewhite AJ, Niezgoda J, et al. Oxidized Regenerated Cellulose/Collagen Dressings: review of evidence and recommendations. Adv Skin Wound Care 2017;30:1-8.

49. Han G, Ceilley R. Chronic Wound Healing: a review of current management and treatments. Adv Ther 2017;34:599-610.

\section{Abbreviations and Acronyms}

AOTF-PMT = acousto-optic tunable filter/ photomultiplier tube

$\mathrm{CaO}=$ Calcium Oxide

$\mathrm{CCM}=$ complete culture medium

$\mathrm{CLSM}=$ confocal laser scanning microscopy

$\mathrm{DFU}=$ diabetic foot ulcer

DMEM = Dulbecco's modified Eagle's mediumF

$\mathrm{FBS}=$ fetal bovine serum

FE-SEM = field emission scanning electron microscopy

$H \& E=$ Hematoxylin and Eosin

$\mathrm{IR}=$ ischemia/reperfusion

$\mathrm{Na}_{2} \mathrm{O}=$ Sodium Oxide

$\mathrm{PBS}=$ phosphate-buffered saline

PLA $=$ poly(lactic acid)

PMN = polymorphonuclear leukocytes

$\mathrm{P}_{2} \mathrm{O}_{5}=$ phosphorus pentoxide

TFE $=$ 2,2,2-trifluoroethanol

$\mathrm{Ti}_{2} \mathrm{O}=$ Titanium Oxide 


\section{AUTHOR QUERY FOR WOUND-2020-1206-VER9-AMODIO_1P}

AU1: Please identify (highlight or circle) all authors' surnames for accurate indexing citations.

AU2: "Biomaterials for Regenerative Therapies Group" is mentioned in corresponding author's address, but not mentioned in author's affiliation. Please check.

AU3: Please check the hierarchy of heading levels in the article.

AU4: "Supplementary Figs. S1 and S2" have been changed to "Supplementary Videos S1 and S2." Please check

AU5: Please provide the degree abbreviation of the author "Mercè Alsina-Gibert" in "ABOUT THE AUTHORS" section.

AU6: Earlier Ref. 10 was duplicate entry of Ref. 7. So Ref. 10 has been deleted and renumbered from 11 onward. AU7: Please provide the title for "Table 2."

AU8: Figure 1 is not cited in the text. Please insert the citation in the text.

AU9: "+" is not found in the artwork of "Figure 5" and "*" is not mentioned in the caption of "Figure 5." Please check.

AU10: “*” and “***” are not mentioned in the caption of "Figure 6." Please check. 